\title{
UV absorption cross sections of vaporized 1-methylnaphthalene at elevated temperatures
}

\author{
Ulrich Retzer ${ }^{1,2} \cdot$ Hannah Ulrich ${ }^{1,2} \cdot$ Florian J. Bauer $^{1,2} \cdot$ Stefan Will ${ }^{1,2} \cdot$ Lars Zigan $^{1,2}$
}

Received: 20 November 2019 / Accepted: 12 February 2020 / Published online: 2 March 2020

(c) The Author(s) 2020

\begin{abstract}
In this work, the UV absorption cross sections of the fluorescence tracer 1-methylnaphthalene are determined in the range of 230-330 nm. The experiments are performed in a continuously scavenged gas flow cell, which allows for defined homogeneous conditions regarding temperature, pressure, and tracer/fuel composition. A LSDS (laser driven light source) is used for irradiation, which enables high spectral emission intensities in the UV range studied. For detection, a spectrograph in combination with an intensified camera is applied. Absorption cross sections at temperatures up to $850 \mathrm{~K}$ are determined and compared to sparsely available published data. Possible uncertainties caused by the optical setup and the flow cell, respectively, are considered.
\end{abstract}

\section{Introduction}

Polycyclic aromatic hydrocarbons (PAH) such as 1-methylnaphthalene (1-MN) play an important role in the field of combustion technology. As a soot precursor, the experimental investigation of PAH can contribute to understand the soot formation process. As both PAH and soot have a high toxicological potential, the concentration in the flue gas should be kept as low as possible, also in order to meet the required emission limit values. Absorption spectroscopy is a suitable measurement technique for the investigation of optical properties of PAH. Several PAH, including naphthalene, have already been studied by using UV-VIS absorption spectroscopy [1]. Additionally, PAH such as 1-MN [2-6], 2-MN [4], naphthalene [6-8], dimethylnaphthalene [8], anthracene [9], and perylene [10] are applied as laser induced fluorescence (LIF) tracers for mixing studies

Electronic supplementary material The online version of this article (https://doi.org/10.1007/s00340-020-7400-z) contains supplementary material, which is available to authorized users.

Lars Zigan

lars.zigan@fau.de

1 Lehrstuhl für Technische Thermodynamik (LTT), FriedrichAlexander-Universität Erlangen-Nürnberg (FAU), Am Weichselgarten 8, 91058 Erlangen, Germany

2 Erlangen Graduate School in Advanced Optical Technologies (SAOT), Friedrich-Alexander-Universität Erlangen-Nürnberg (FAU), Paul-Gordan-Straße 6, 91052 Erlangen, Germany in gas flows and combustion processes. The LIF technique can be used for the determination of temperature and fuel distribution, thus contributing to the optimization of the combustion process and to the reduction of pollutant emissions. To enable a quantitative determination of the parameters of interest, a calibration of the fluorescence behavior of the tracer is necessary. Since the fluorescence signal is directly proportional to the absorption cross section $\sigma_{\text {abs }}$, this quantity must be determined to gain a precise understanding of the fluorescence characteristics. For 1-MN only few and inconsistent UV absorption data is available and further experiments are necessary for quantitative prediction and modeling of fluorescence emissions. For example, Suto et al. [11] studied, among others, the absorption behavior of naphthalene and its derivatives 1-MN and 2-ethylnaphthalene from 190 to $295 \mathrm{~nm}$ at $297 \mathrm{~K}$ using synchrotron radiation. For $1-\mathrm{MN}$ a value of $\sim 1.8 \cdot 10^{-17} \mathrm{~cm}^{2}$ at $266 \mathrm{~nm}$, which is the typical excitation wavelength for aromatic LIF tracers, is given. Orain et al. [8] showed a non-monotonic function of the temperature-dependent absorption cross section of naphthalene from 350 to $900 \mathrm{~K}$ at $266 \mathrm{~nm}$. For naphthalene and its derivatives 1-MN and 1,3-dimethylnaphthalene a value of $1.28 \cdot 10^{-17} \mathrm{~cm}^{2}$ is given at $350 \mathrm{~K}$ and $266 \mathrm{~nm}$. Baranger [12] showed nearly temperature independent values of $7.2 \cdot 10^{-18} \mathrm{~cm}^{2}$ for naphthalene, $7.5 \cdot 10^{-18} \mathrm{~cm}^{2}$ for 1,3-dimethylnaphthalene, and $4.1 \cdot 10^{-18} \mathrm{~cm}^{2}$ for $1-\mathrm{MN}$ from 440 to $620 \mathrm{~K}$ at $266 \mathrm{~nm}$. It should be noted that these values are one order of magnitude lower than in the other two references $[8,11]$. 
The fluorescence signals of 1-MN have been calibrated in a wide pressure and temperature range for varying mixtures with oxygen. This calibration data serves for the determination of temperature and fuel distribution using 2-color LIF and FAR-LIF (fuel-air ratio) [3, 4, 6, 13]. However, no detailed temperature-dependent analysis of the absorption behavior has been performed yet, which is necessary to explain the observed non-monotonous fluorescence calibration functions [3]. For this purpose, the temperature dependent absorption cross section of 1-MN is analyzed from 350 to $850 \mathrm{~K}$ in a spectral range of $230-330 \mathrm{~nm}$ in this experimental study.

\section{Experiment}

The experiments are performed in a continuously scavenged flow cell ( $\mathrm{HTC}^{2}$-high temperature calibration cell) at homogenous conditions (Fig. 1).

To allow evaporation of the tracer at temperatures as low as $350 \mathrm{~K}$ and to avoid complete absorption of the light, a gas mixture of 1 vol. \% 1-MN (Merck Millipore, purity $\geq 94 \%$ ) in isooctane (Rotisolv ${ }^{\circledR} \mathrm{UV} / \mathrm{IR}$ grade, purity $\geq 99.8 \%$ ) is added to the gas flow entering the cell. The tracer/fuel mixture is adjusted and evaporated by a controlled evaporating and mixing system (CEM, Bronkhorst, not shown in Fig. 1). After the heating section, the carrier gas (dry compressed ambient air) and nitrogen (Linde, nitrogen 5.0, purity 99.999\%) and the vaporized 1-MN/isooctane flow are combined in the mixing section of the cell. The volume flow rate

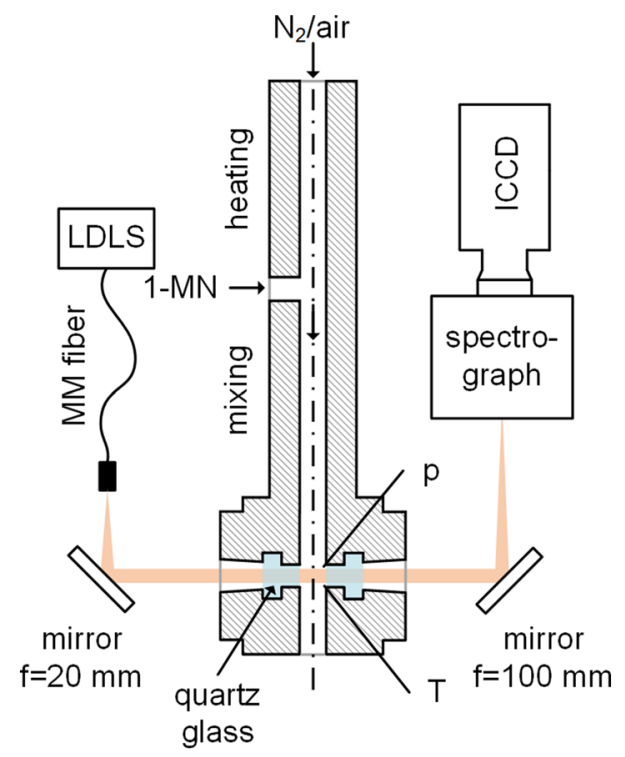

Fig. 1 Optical setup including a schematic cross-sectional view of the $\mathrm{HTC}^{2}$ calibration cell. $M M$ multimode, $L D L S$ Laser-Driven Light Source, $p$ pressure, $T$ temperature of the carrier gas is adjusted by mass flow controllers (MFC, Bronkhorst). During all measurements, the molar concentration of the fuel $/$ tracer is kept constant at $0.5 \mathrm{~mol} / \mathrm{m}^{3}$ in the measurement volume. Depending on the measurement point, the residence time of the mixture at elevated temperature varies between 116 and $283 \mathrm{~ms}$. The residence time is calculated from the known total volume flow rate and the distance between the mixing zone and the center of the LIF measurement plane in the cell. Specially designed windows and flanges guarantee a constant diameter of the flow duct over the entire measuring volume, thus minimizing cavities that lead to recirculation zones and temperature gradients. The stepped quartz glass windows (Sico, SQ1 quality, transmission of 0.96 at $200 \mathrm{~nm}$ ) have a thickness of $15 \mathrm{~mm}$ and an inner diameter of $7.5 \mathrm{~mm}$. The absorption path between both windows is $24.6 \mathrm{~mm}$. The studied temperature ranges are $350-850 \mathrm{~K}$ being varied in steps of $50 \mathrm{~K}$. Additional experiments using broadband supercontinuum laser absorption spectroscopy (SCLAS) were performed to investigate the fuel/tracer conversion by detection of the produced $\mathrm{H}_{2} \mathrm{O}$ molecule (not shown here, this will be presented in a forthcoming paper). The results do not show any indication of tracer decomposition and oxidation at maximal temperature conditions (equivalence ratio of $\Phi=1$ up to $850 \mathrm{~K}$ ). To be completely sure, for temperatures higher than $500 \mathrm{~K}$, all measurements were performed in pure nitrogen atmosphere. From 350 to $500 \mathrm{~K}$, dry air is used as carrier gas. Control measurements at $500 \mathrm{~K}$ did not show any influence of the carrier gas on the absorption behavior of $1-\mathrm{MN}$. Prior to the experiment, a temperature field analysis in the cell was performed with three thermocouples (type K, TC Direct, $1 \mathrm{~mm}$ ) at a distance of $7 \mathrm{~mm}$ along the absorption path. A maximum temperature gradient of $3.5 \%$ was determined between the three thermocouples at $750 \mathrm{~K}$, while the average temperature gradient over all operation points was $2.1 \%$. An average temperature determined by the three thermocouples is used as reference for the absorption measurements. The pressure was measured with a pressure transducer (PAA-33X/80794, Keller). The pressure is kept constant at $0.4 \mathrm{MPa}$ for all operation points, which corresponds to the minimum pressure at which the required volume flows can still be adjusted. Uncertainties depending on temperature, molar concentration of the tracer as well as the influence of pressure are presented at the end of the manuscript. For irradiation, a Laser-Driven Light Source (LDLS, ENERGETiQ EQ-99X-FC) is used, which provides broadband emission from 190 to $2100 \mathrm{~nm}$. After the fiber exit, the light is first collimated $(6 \mathrm{~mm})$ by an aluminum coated parabolic mirror (Newport, 50328AL), passed through the measuring volume and then focused by a cylindrical concave UV-enhanced aluminum mirror (Thorlabs, CCM254-100-F01) onto the entrance slit of the spectrograph (Acton SP2300, slit: $5 \mu \mathrm{m}$ ). A $3001 / \mathrm{mm}$ grating with a blaze wavelength of $300 \mathrm{~nm}$ is 
applied. The spectrally resolved signal is then detected by an intensified CCD camera (DiCAM pro 12 bit, exposure time: $100 \mu \mathrm{s}$, spectral resolution: $1.15 \mathrm{~nm}$, quantified with a $\mathrm{Hg}(\mathrm{Ar})$-lamp at $253.7 \mathrm{~nm}$ ). Additional measurements are performed using a commercially available spectrometer (Ocean Optics, Ocean FX, CMOS detector, exposure time: $60 \mu \mathrm{s}$, slit: $50 \mu \mathrm{m}$, spectral resolution: $1.08 \mathrm{~nm}$ ) to investigate the effect of the detection system on the results. More information about the LIF-Tracer 1-MN and the calibration cell can be found in references $[3,5,13]$.

For every operation point, six absorption measurements (I) of 377 images each (limited by the internal memory of the camera) are performed to minimize the influence of potential temporal fluctuations and spatial inhomogeneities. Directly before and after these measurements, three reference measurements $\left(I_{0}\right)$ with 377 images each are performed. Out of these images, a mean vector for $I$ and $I_{0}$ covering the spectral range considered is calculated within a region of interest (ROI). The ROI has a height of 50 pixels, covering the main part of the beam diameter. $I_{0}$-measurements are done at the same conditions as the $I$-measurements but in pure air/ nitrogen without the fuel/tracer flow. The absorption cross section is then determined based on these $I / I_{0}$-vectors using the Beer-Lambert law. This procedure is repeated at three different days. Additional $I_{0}$-measurements with isooctane at the same molar concentration as in the $I$-measurements did not show any influence on the determined absorption spectrum in the relevant spectral range of $230-330 \mathrm{~nm}$.

\section{Results}

All the results presented below show the mean value of the three measurements (each consisting of six individual measurements) performed at three different days. Figure 2

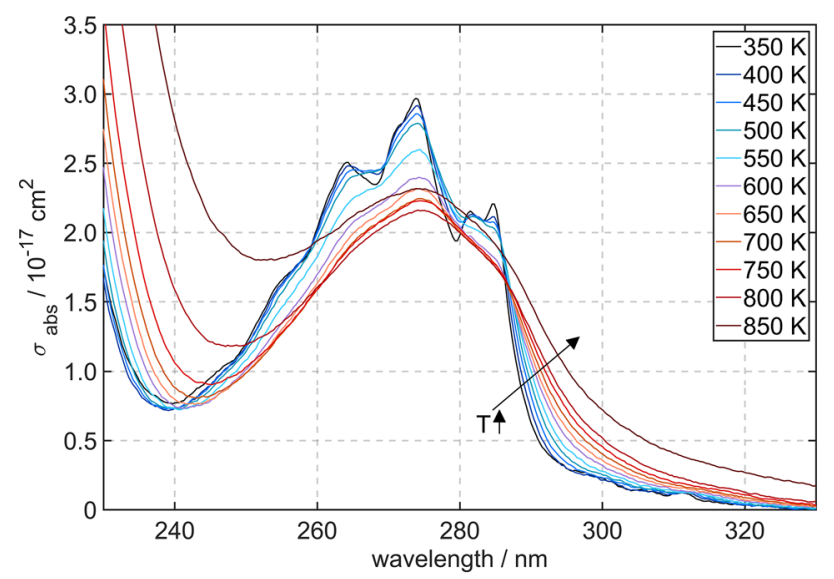

Fig. 2 Temperature-dependent absorption spectra of $1-\mathrm{MN}$ at $0.4 \mathrm{MPa}$ exhibits the temperature dependent absorption spectra of $1-\mathrm{MN}$ at $0.4 \mathrm{MPa}$ between 350 and $850 \mathrm{~K}$ in the range of 230-330 nm.

At $350 \mathrm{~K}$, clear spectral structures with three peaks at $264 \mathrm{~nm}, 274 \mathrm{~nm}$, and $285 \mathrm{~nm}$ are visible. A local maximum of the absorption cross section of about $3 \cdot 10^{-17} \mathrm{~cm}^{2}$ is found at $350 \mathrm{~K}$ and $274 \mathrm{~nm}$. With increasing temperature, the structures are less distinct and the spectra are broadened and red-shifted. According to the Boltzmann distribution, the probability of reaching higher vibrational levels within the electronic ground state increases with increasing temperature. Therefore, the energy gap between the singlet states $S_{0} \rightarrow S_{1}$ decreases, which can explain the spectral red-shift of the absorption spectra with increasing temperature. The convolution of each spectrum with the underlying Maxwell-Boltzmann distribution kernel for elevated temperature smears out single peaks and the spectra become less structured. With increasing temperature, more vibrational and rotational levels are occupied. Additionally, the energy gap between vibrational levels decreases as the vibrational level increases with temperature. Furthermore, Doppler broadening increases with temperature. At temperatures higher than $800 \mathrm{~K}$ the "red part" of the $\mathrm{S}_{0} \rightarrow \mathrm{S}_{2}$ transition $(<240 \mathrm{~nm})$ could be significant and thus overlaps with the $\mathrm{S}_{0} \rightarrow \mathrm{S}_{1}$ transition. This would lead to increasing absorption cross-sections in the considered spectral range from 230 to $330 \mathrm{~nm}$. Increasing absorption cross-sections at high temperatures were also found for naphthalene [8] (as discussed below) and toluene [14].

The spectral shift leads to increased absorption cross sections with increasing temperature at wavelengths above $\sim 287 \mathrm{~nm}$. The peak maximum slightly shifts from $274 \mathrm{~nm}$ at $350 \mathrm{~K}$ to $275 \mathrm{~nm}$ at $800 \mathrm{~K} / 850 \mathrm{~K}$. Less distinct spectral features and spectral broadening with increasing temperature were also observed for naphthalene [15]. Compared to naphthalene, a red-shift of the 1-MN absorption spectra of approximately five nanometers is found (at constant temperature). In general, the absorption spectrum of 1-MN shows less distinct structure than that of naphthalene $[11,15]$. Since $1-\mathrm{MN}$ is usually excited at $266 \mathrm{~nm}$ in tracer-LIF measurements [3-5, 13], Fig. 3 shows the course

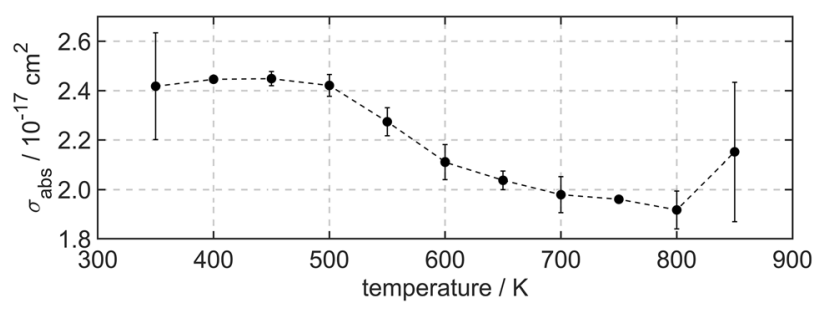

Fig. 3 Temperature-dependent absorption cross section of 1-MN including the $95 \%$ confidence interval at $266 \mathrm{~nm}$. Line is inserted to guide the eye 
of the absorption cross section with the temperature at this wavelength.

From 350 to $800 \mathrm{~K}$ the peak maximum at $274 \mathrm{~nm}$, shown in Fig. 2, decreases. However, due to the above-mentioned less distinct structures with increasing temperature, the absorption cross section at $266 \mathrm{~nm}$ stays almost constant between 350 and $500 \mathrm{~K}$. A maximum cross section of $2.45 \cdot 10^{-17} \mathrm{~cm}^{2}$ is found at $450 \mathrm{~K}$. From 500 to $800 \mathrm{~K}$, the absorption cross section decreases by about $20 \%$. From 800 to $850 \mathrm{~K}$, the mean value of the absorption cross-section increases again. The large error bars at $850 \mathrm{~K}$ already indicate that this non-monotonous behavior cannot be clearly proven. The absorption cross section increases with a probability of $62 \%$. However, a possible physical reason is given above and similar results were already shown for naphthalene: there, due to the spectral features, the absorption cross section first increases up to $500 \mathrm{~K}$, then decreases up to $850 \mathrm{~K}$, and then increases again from 850 to $900 \mathrm{~K}$ [8]. In contrast to this result, Zhang et al. [16] showed a linear increase of the absorption cross section of naphthalene at $266 \mathrm{~nm}$ from 673 to $1373 \mathrm{~K}$.

The error bars exhibited in Fig. 3 correspond to the 95\% confidence interval over three values determined on three different days. The confidence interval was calculated using the student's $t$-distribution ( $t$-value: $4.303,2.48$ ). Between 400 and $800 \mathrm{~K}$, the individual measurements were very similar with a deviation of $2.2 \%$. At $350 \mathrm{~K}$ and $850 \mathrm{~K}$, the 2.48 value is significantly higher with values of $9.0 \%$ and $13.1 \%$, respectively. The increased standard deviation at $350 \mathrm{~K}$ may be explained by a higher variation of the tracer concentration as the fuel/tracer mixture cannot be completely evaporated in the CEM-system (due to the low vapor pressure). At $850 \mathrm{~K}$, the calibration cell operates at its flow limit, which can cause fluctuations of the mass flow and inhomogeneities. The standard deviation at $266 \mathrm{~nm}$ is a representative value for all measurement points.

In the following, an error estimation will be given including a comparison with literature data and a discussion about the influence of the detection system on the results. Figure 4 shows the result of this work at $350 \mathrm{~K}$ in comparison to the available literature data of Suto et al. [11] in the range of $230-295 \mathrm{~nm}$ at $297 \mathrm{~K}$ and Orain et al. [8] at $266 \mathrm{~nm}$ and at $350 \mathrm{~K}$.

A comparison of the absorption spectrum with data of Suto et al. shows a good agreement regarding the positions of the three spectral features (spectral shift at $274 \mathrm{~nm}: \sim 0.5 \mathrm{~nm}$ ) and the relative height of the individual peaks. The relative height of the peaks in Suto et al. is slightly increased and the peaks are more distinct, which could be explained by the lower temperature. In the present study, measurements below $350 \mathrm{~K}$ have not been possible as the CEM-system does not enable complete evaporation of the fuel/tracer mixture due to the absolute pressure of

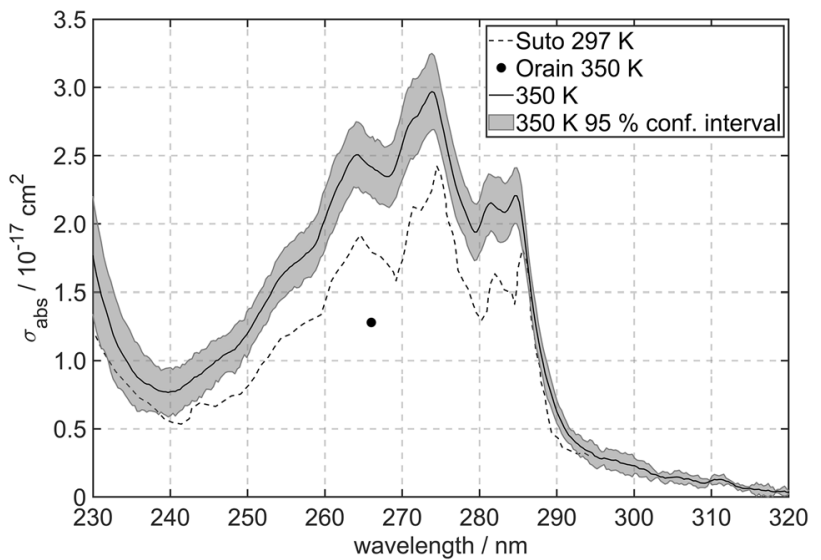

Fig. 4 Absorption cross sections including the 95\% confidence interval in comparison to available literature data (dashed line-[11], $\operatorname{dot}-[8])$

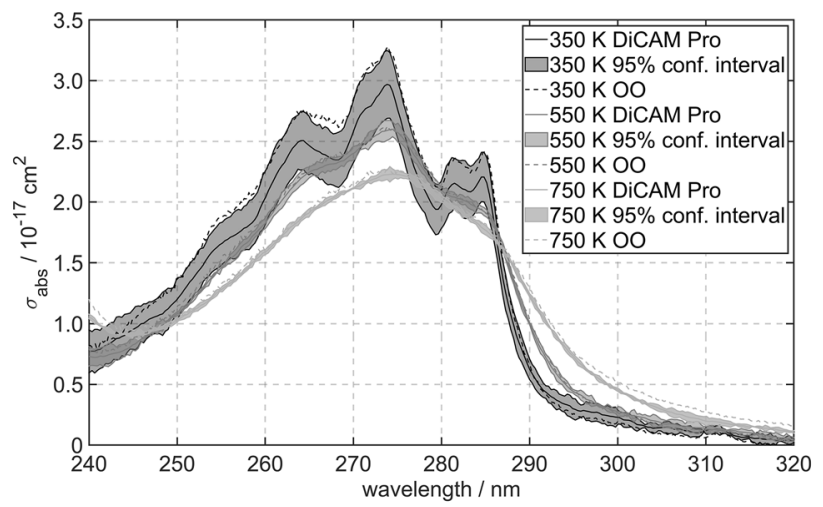

Fig. 5 Absorption cross sections [mean value and 95\% confidence interval) from 240 to $320 \mathrm{~nm}$ at $350 \mathrm{~K}, 550 \mathrm{~K}$, and $750 \mathrm{~K}$ determined by two detection systems (solid line: Acton SP2300+DiCAM Pro, dashed line: Ocean Optics FX (OO)]

$0.4 \mathrm{MPa}$ (Suto et al.: $<1.33 \cdot 10^{-6} \mathrm{MPa}$ ). Between 230 and $286 \mathrm{~nm}$, the absolute values of this work are higher than those reported by Suto et al. (at $274 \mathrm{~nm} \sim 0.5 \cdot 10^{-17} \mathrm{~cm}^{2}$ ). Between 286 and $295 \mathrm{~nm}$, a good agreement of the absorption cross sections is found. Orain et al. [8] determined a value of $1.28 \cdot 10^{-17} \mathrm{~cm}^{2}$ at $350 \mathrm{~K}$ and $266 \mathrm{~nm}$ for $1-\mathrm{MN}$, naphthalene and 1,3-dimethylnaphthalene, which is significantly lower than the value of this work $\left(2.4 \cdot 10^{-17} \mathrm{~cm}^{2}\right)$. It should be noted that, due to significant differences in the spectral structure, it is unlikely that all three substances have exactly the same absorption cross section under the same conditions.

Additionally, the deviation in absorption cross sections introduced by the detector is considered. Figure 5 shows a comparison of two detection systems (Acton SP2300 + DiCAM Pro and Ocean Optics FX) at $350 \mathrm{~K}$, $550 \mathrm{~K}$, and $750 \mathrm{~K}$. 
Table 1 Error sources

\begin{tabular}{ll}
\hline Error source & \\
\hline MFC air/nitrogen & $2.5 \%$ (Manufacturer specifications) \\
CEM system & $3.2 \%$ (Manufacturer specifications) \\
Pressure transducer & $1.2 \%$ (Manufacturer specifications) \\
Thermocouple & $0.7 \%$ (Manufacturer specifications) \\
Light source & $<1.0 \%$ (95\% Confidence interval, measured) \\
\hline
\end{tabular}

For both detection systems, the optical setup and the experimental parameters were the same. For all temperatures the spectral position and shape is almost identical. For $550 \mathrm{~K}$ and $750 \mathrm{~K}$ also the absolute values of the absorption cross sections are hardly influenced by the detection system. At $350 \mathrm{~K}$ for wavelengths up to $286 \mathrm{~nm}$, the absolute values determined by the ocean optics spectrometer are slightly increased. At $274 \mathrm{~nm}$, the absorption cross sections determined by $\mathrm{OO}$ are $9 \%$ higher. However, as discussed above, the uncertainty at this temperature is also increased. We conclude that the deviations are mainly introduced by the flow cell, which lead to large variations. The spectral resolution of the Acton SP2300 + DiCAM-system is reduced by the intensifier. This results in almost the same resolution as the OO-system despite the much smaller slit size ( $5 \mu \mathrm{m}$ in comparison to $50 \mu \mathrm{m}$ for OO). The comparison shows that the detection chip (ICCD or uncooled CMOS) does not influence the results. Furthermore, the influences of fuel/tracer concentration and the absolute pressure are investigated (not shown here). Basically, these parameters variations could introduce differences in the flow and mixing behavior in the cell. The pressure influence on the results is negligibly low. The absorption cross sections determined at 1.2 MPa differ on average by $1.6 \%$ from the results at $0.4 \mathrm{MPa}$ (at $274 \mathrm{~nm}$ and $500 \mathrm{~K})$. By reducing the molar concentration of the fuel/ tracer from $0.5 \mathrm{~mol} / \mathrm{m}^{3}$ to $0.375 \mathrm{~mol} / \mathrm{m}^{3}$, the results differ by $2.6 \%$ (at $274 \mathrm{~nm}$ and $500 \mathrm{~K}$ ), which is lower than the maximum error for the MFC-adjusted tracer concentration in the cell (see below).

Finally, an estimate on the possible main sources of error is given. Errors are introduced either by the components of the flow cell itself or by the optical setup. The MFCs for adjusting the air/ $\mathrm{N}_{2}$ volume flow, the CEM system and the temperature as well as pressure transducer and the fluctuation of the LDLS contribute to a maximum error of $4.4 \%$ for the absorption cross section. This error was calculated by means of an error propagation. The individual uncertainties are provided in Table 1. The fluctuation of the LDLS is $1.0 \%$ (mean value of the $95 \%$ confidence interval of 18 (six measurements on 3 days each) averaged $I_{0}$ vectors in the considered spectral range from 230 to $330 \mathrm{~nm}$. One $I_{0}$ vector consists of 377 reference measurements without tracer flow but gas flow at $0.4 \mathrm{MPa}$ and at $350 \mathrm{~K}$. To minimize the influence of the LDLS fluctuations, all signals $I$ and $I_{0}$ are normalized to the signal intensity at $\sim 390 \mathrm{~nm}$ (as no absorption takes place at this wavelength). In addition, the temperature gradient along the absorption path must be taken into account. Here, between 350 and $850 \mathrm{~K}$, an average temperature gradient of $2.1 \%$ between the three thermocouples was found (as mentioned above). The linearity of the camera is not taken into account as the absorption signal $I$ is normalized to the reference signal $I_{0}$.

\section{Conclusion}

In this work, the absorption cross sections of 1-MN from 230 to $330 \mathrm{~nm}$ were determined for temperatures from 350 to $850 \mathrm{~K}$. A laser driven light source was used for broadband emission, which provides high signal intensities in the UV range. A spectrograph in combination with an ICCD camera was applied as detection system. With increasing temperature, characteristic features within the absorption spectra of 1-MN become less distinct and the spectra are broadened and red-shifted. At $266 \mathrm{~nm}$, the absorption cross section nearly stays constant between 350 and $500 \mathrm{~K}$ and then decreases up to $800 \mathrm{~K}$. Finally, it tends to increase again from 800 to $850 \mathrm{~K}$, which could be explained by an overlap of the of the $S_{0} \rightarrow S_{2}$ transition $(<240 \mathrm{~nm})$ with the $S_{0} \rightarrow S_{1}$ transition. A comparison with the results of a commercially available spectrometer showed only small deviations. An error estimation was conducted, which showed that the components of the flow cell introduced the largest uncertainty of the absorption cross sections.

The work can help for an improved understanding of LIF calibration data of the tracer 1-MN for the investigation of the mixture formation, e.g., in diesel combustion processes. Furthermore, 1-MN as a soot precursor in reactive flows can be investigated using LIF and absorption spectroscopy with the help of this work and contribute to the understanding of the soot formation process.

Acknowledgements Open Access funding provided by Projekt DEAL.

Funding This study was funded by Deutsche Forschungsgemeinschaft (DFG Zi 1384/3) and Erlangen Graduate School of Advanced Optical Technologies (SAOT).

\section{Compliance with ethical standards}

Conflict of interest The authors declare that they have no conflict of interest.

Open Access This article is licensed under a Creative Commons Attribution 4.0 International License, which permits use, sharing, adaptation, distribution and reproduction in any medium or format, as long as you give appropriate credit to the original author(s) and the source, provide a link to the Creative Commons licence, and indicate if changes 
were made. The images or other third party material in this article are included in the article's Creative Commons licence, unless indicated otherwise in a credit line to the material. If material is not included in the article's Creative Commons licence and your intended use is not permitted by statutory regulation or exceeds the permitted use, you will need to obtain permission directly from the copyright holder. To view a copy of this licence, visit http://creativecommons.org/licenses/by/4.0/.

\section{References}

1. A. Tregrossi, B. Apicella, A. Ciajolo, C. Russo, Chem. Eng. Trans. 57, 1447 (2017)

2. F. Payri, J.V. Pastor, J.M. Pastor, J.E. Juliá, Int. J. Eng. Res. 7, 77 (2006)

3. U. Retzer, W. Fink, T. Will, S. Will, L. Zigan, Appl. Phys. B 125, 124 (2019)

4. J. Trost, L. Zigan, A. Leipertz, D. Sahoo, P.C. Miles, Appl. Opt. 52, 8001 (2013)

5. J. Trost, L. Zigan, A. Leipertz, D. Sahoo, P.C. Miles, Int. J. Eng. Res. 15, 741 (2014)

6. S.A. Kaiser, M.B. Long, Proc. Combust. Inst. 30, 1555 (2005)

7. S. Faust, G. Tea, T. Dreier, C. Schulz, Appl. Phys. B 110, 81 (2013)
8. M. Orain, P. Baranger, B. Rossow, F. Grisch, Appl. Phys. B 102, $163(2011)$

9. J.K. Yoon, K.J. Myong, J. Senda, H. Fujimoto, J. Mech. Sci. Technol. 23, 2565 (2009)

10. F.P. Hindle, T.L. Yeo, K.B. Ozanyan, N.R.J. Poolton, H. McCann, IEEE Sens. J. 3, 766 (2003)

11. M. Suto, X. Wang, J. Shan, L.C. Lee, J. Quant. Spectrosc. Radiat. Transf. 48, 79 (1992)

12. P. Baranger, Kerosene Detection Using Laser Induced Fluorescence Imaging for Aeronautical Engines Application (Université Paris Sud, Paris, 2004)

13. S. Lind, U. Retzer, S. Will, L. Zigan, Proc. Combust. Inst. 36, 4497 (2017)

14. W. Koban, J.D. Koch, R.K. Hanson, C. Schulz, Phys. Chem. Chem. Phys. 6, 2940 (2004)

15. H. Grosch, Z. Sárossy, H. Egsgaard, A. Fateev, J. Quant. Spectrosc. Radiat. Transf. 156, 17 (2015)

16. Y. Zhang, L. Wang, P. Liu, Y. Li, R. Zhan, Z. Huang, H. Lin, Appl. Phys. B 125, 6 (2018)

Publisher's Note Springer Nature remains neutral with regard to jurisdictional claims in published maps and institutional affiliations. 